\title{
Enterprise 2.0 - Gegenwart und Zukunft
}

\section{Vorschlag einer Forschungsagenda}

\author{
Alexander Richter', Angelika C. Bullinger ${ }^{2}$ \\ ${ }^{1}$ Forschungsgruppe Kooperationssysteme, Universität der Bundeswebr München \\ ${ }^{2}$ Lehrstubl für Wirtschaftsinformatik I, Universität Erlangen-Nürnberg
}

\section{Einleitung}

Im Angesicht einer stark im Wandel begriffenen Unternehmenswelt - Globalisierung, verkürzte Produktlebenszyklen, stark steigende Anforderungen an Mobilität und Flexibilität der Mitarbeiter - gewinnt die IT-gestützte Zusammenarbeit weiter an Bedeutung. In diesem Zusammenhang erscheint zwar eine "Groupware Suite", welche die notwendige Sicherheit und Orientierung zu bieten in der Lage wäre, oberflächlich als Lösung. Allerdings zeigt sich, dass die Entwicklung eines umfassenden Instrumentenkastens zur Unterstützung von Koordination, Kommunikation und Kollaboration nicht ohne weiteres möglich ist. Stattdessen fanden in den letzten Jahren insbesondere verschiedene modulare Instrumente, die im so genannten „Web 2.0“ privat außergewöhnlich stark genutzt wurden, auch in der Unternehmenspraxis zunehmend Anwendung.

Das Web 2.0 bringt neue Paradigmen mit sich: So haben sich die Benutzer beispielsweise von reinen Konsumenten zu Produzenten entwickelt, die Inhalte nicht mehr nur empfangen, sondern diese selbst bereitstellen, editieren, bewerten und kommentieren. (z.B. Zerfaß et al. 2009). Diesen Paradigmenwandel gilt es nun auf Firmenebene in angemessener Form ebenfalls zu vollziehen und infolgedessen stellt sich die Frage nach den Einsatzmöglichkeiten des „Web 2.0 in Unternehmen“ (Back et al. 2009) bzw. des „Enterprise 2.0“ (Koch und Richter 2009). Dabei steht der von Andrew McAfee (2006) geprägte Begriff Enterprise 2.0 in diesem Beitrag für das große Potential von Web 2.0-Technologien bzw. Social Software, wie Wikis und Weblogs, zur Unterstützung der unternehmensinternen und übergreifenden Zusammenarbeit.

Im Gegensatz zum privaten Internet, das eher durch informelle Strukturen gekennzeichnet ist (Jahnke 2009), müssen die Beteiligten in den Unternehmen allerdings verschiedene unternehmensspezifische Herausforderungen meistern, wie z.B. die Einbeziehung von Organisationsstrukturen und -Prozessen. Diese und andere Spezifika sollten bei der soziotechnischen Systemgestaltung der Werkzeuge be- 
rücksichtigt werden. In den vergangenen Jahren wurde der Einsatz von Social Software in Unternehmen deswegen in zahlreichen Forschungsprojekten untersucht. und es findet sich ein großer Forschungskörper. Dieser reicht von Einzelund Mehrfachfallstudien über Aktionsforschung zu quantitativen Umfragen. Dabei finden sich Erkenntnisse zur Verbreitung einzelner Anwendungsklassen, Nutzenanalysen, oder auch die Identifikation von Barrieren und Erfolgsfaktoren von Enterprise 2.0. Angesichts dieser facettenreichen Entwicklung scheint Orientierung notwendig geworden zu sein. Neben einer Aufbereitung des aktuellen Stands der Forschung schlägt der vorliegende Beitrag sechs Metathemata vor, welche Querverbindungen über die einzelnen Forschungsansätze herzustellen in der Lage sind. Er zielt auf die Identifikation zukünftig relevanter Forschungsfelder im Bereich Enterprise 2.0 und zeigt somit eine Forschungsagenda für das Feld auf.

Zweifellos lässt sich sagen, dass die Unternehmenskultur v.a. aufgrund der flachen Hierarchien im Enterprise 2.0 eine große Rolle spielt. Deswegen werden sowohl von der Forschung als auch in der Praxis (z.B. Bughin et al. 2008) regelmäBig erhebliche Unterschiede (u.a. in den Nutzungspraktiken) insbesondere auch zwischen verschiedenen Kulturen (wie USA und Deutschland) festgestellt. In Anbetracht dieser Unterschiede, die jedoch nicht Gegenstand der weiteren Analyse sind, beschränken wir uns mit unseren Aussagen auf den deutschsprachigen Raum. Wir beginnen mit einer Aufarbeitung der deutschsprachigen Literatur im Gebiet (Abschnitt 2). Danach werden die Ergebnisse eines eintägigen Workshops zur Zukunft von Enterprise 2.0 mit 19 Experten aus Wissenschaft und Praxis vorgestellt (Abschnitte 3 und 4). Zusammenfassung und Diskussion schließen den Beitrag.

\section{Literaturüberblick: Gegenwart von Enterprise 2.0}

Um den aktuellen Stand der Veröffentlichungen umfassend zu bewerten, haben wir eine systematische Literaturrecherche durchgeführt. Der Prozess der Datenerhebung für die Literaturrecherche bestand aus drei getrennten Aktivitäten: (1) Schlagwort-Suche „Enterprise 2.0“ (138 Treffer) und „Social Software im/in Unternehmen“" (zusammen 118 Treffer) in der deutschen Version von Google Scholar, (2) Analyse der Literaturverzeichnisse in den identifizierten Quellen sowie (3) Hinweise von kooperierenden Forschern ${ }^{1}$. Die Anzahl der identifizierten Veröffentlichungen (>300) wurde folgendermaßen eingeschränkt: Es wurden ausschließlich (a) empirische Studien, die (b) wie oben erläutert im deutschsprachigen Raum² und (c)

\footnotetext{
1 Aufgrund der Neuartigkeit des Themas scheint die Berücksichtigung von Arbeiten notwendig, die von kooperierenden Forschern empfohlen wurden. Diese wurden zur Mitarbeit an der folgenden Wiki-Seite aufgerufen: http://wiki.informatik.unibw-muenchen.de/Main/Enterprise2Studien.

2 Eine Ausnahme stellen die Studien von Bughin et al. dar. Diese wurden aufgenommen, weil sie aufgrund der differenzierten Darstellung auch für den deutschsprachigen Raum wesentliche Aussagenkraft haben.
} 
primär erhoben ${ }^{3}$ wurden, berücksichtigt, die sich (d) auf den inner- und zwischenbetrieblichen Einsatz von Social Software zur Zusammenarbeit beziehen ${ }^{4}$. Diese Auswahl wurde von drei weiteren im Bereich tätigen Forschern überprüft und punktuell erweitert.

In der Literatur lassen sich sowohl quantitative Studien identifizieren, die mit der Hilfe von Unternehmensbefragungen einen Überblick zum Stand der Nutzung von Social Software in Unternehmen vermitteln als auch qualitative und quantitative Studien, die ausgehend von einzelnen Unternehmen Herausforderungen identifizieren und Lösungsvorschläge erarbeiten.

Bei den quantitativen Erhebungen zur Nutzung lassen sich drei Hauptrichtungen erkennen. Es wurde zum einen das Potential von Enterprise 2.0 für einzelne Brachen (z.B. die Finanzbranche (Wittman 2008)) oder einzelne funktionale Ebenen in einem Unternehmen (beispielsweise After Sales Service (Bughin und Manyika 2007), Forschung und Entwicklung (Herrmann 2007) oder Logistik (BITKOM 2007)) untersucht. Desweiteren wurde zwischen dem Einsatz auf Team-Ebene oder im ganzen Unternehmen unterschieden (Leibhammer 2008). Schließlich wurde in den Erhebungen das Interesse der befragten Unternehmen an einzelnen Anwendungsklassen abgefragt (Bughin et al. 2008), die Meinung zu verschiedenen Nutzungsbarrieren (Bughin et al. 2009) oder allgemein das Potential der Anwendungsklassen für bestimmte Nutzungsszenarien (z.B. Berlecon Research 2007; TSystems 2008).

Es liegt in der Natur dieser quantitativen (unpersönlichen) Studien, dass die Erfahrung der Nutzer sowie die tatsächliche Nutzung von Social Software nicht überprüfbar sind. Ferner ist die Berücksichtigung des Einsatzkontextes schwer möglich und es existieren oftmals völlig unterschiedliche Begriffsverständnisse, so dass die Studien nur schwer vergleichbar sind.

Bei den qualitativen (insbesondere Fallstudien) und quantitativen Studien, die sich auf bestimmte Unternehmen beqiehen werden dagegen konkrete Herausforderungen und Lösungsvorschläge im Einsatz- bzw. Unternehmenskontext betrachtet. Dabei erleichtert die Betrachtung greifbarer Anwendungsfälle ein besseres Verständnis für einzelne Faktoren aus der Vielzahl betrachteter Themata.

Die nachfolgende Tabelle soll einen Überblick über die Spannweite der Forschungsarbeiten geben. Aufgrund der hohen Anzahl der Studien wird von jedem Forscher beispielhaft nur eine Arbeit genannt. Ein „*“ stellt einen Hinweis auf die Existenz weiterer, vergleichbarer Arbeiten dar. Die letzte Spalte („Meta“) wird in Abschnitt 5 erläutert.

\footnotetext{
${ }^{3}$ Es fanden also keine Meta-Studien über quantitative (Fuchs-Kittowski et al. 2009) oder qualitative Studien (Granitzer und Tochtermann 2009) Berücksichtigung.

${ }^{4}$ Nicht betrachtet wurden folglich Studien, die sich beispielsweise auf den Einsatz von Social Software zur Kundenbindung beziehen (wie z.B. Döbler 2007).
} 
Tabelle 1: Literaturüberblick zu Enterprise 2.0

\begin{tabular}{|c|c|c|c|c|}
\hline Quelle & $\begin{array}{l}\text { Betrachtete } \\
\text { Themata }\end{array}$ & $\begin{array}{l}\text { Empirische } \\
\text { Basis }\end{array}$ & $\begin{array}{l}\text { Anwendungs- } \\
\text { klasse }\end{array}$ & $\begin{array}{l}\text { Me- } \\
\text { ta }\end{array}$ \\
\hline $\begin{array}{l}\text { Back et al. } \\
(2009)\end{array}$ & $\begin{array}{l}\text { Funktionen, Pro- } \\
\text { duktivität, U.-kultur } \\
\text { und- kontext, Nut- } \\
\text { zerbeteiligung, u.v.a. }\end{array}$ & $\begin{array}{l}8 \text { Einzelfallstudi- } \\
\text { en, Unternehmen } \\
\text { (U.) aller Größen }\end{array}$ & $\begin{array}{l}\text { Wiki, Weblog, } \\
\text { Social Tagging, } \\
\text { SNS }\end{array}$ & $\begin{array}{l}1,2, \\
3,4, \\
5,6\end{array}$ \\
\hline $\begin{array}{l}\text { Benlian et } \\
\text { al. (2009) }\end{array}$ & $\begin{array}{l}\text { Selbstorganisation, } \\
\text { U.-kultur, u.a. }\end{array}$ & $\begin{array}{l}2 \text { Einzelfallstudi- } \\
\text { en in Konzernen }\end{array}$ & $\begin{array}{l}\text { Wiki, Social } \\
\text { Networking } \\
\text { Service (SNS) }\end{array}$ & 3,5 \\
\hline $\begin{array}{l}\text { Blaschke } \\
(2008)^{*}\end{array}$ & $\begin{array}{l}\text { Maße zur Analyse } \\
\text { von Wikis }\end{array}$ & $\begin{array}{l}\text { Einzelfallstudie } \\
\text { in KMU }\end{array}$ & Wiki & 2,6 \\
\hline $\begin{array}{l}\text { Böhringer } \\
\text { et al. (2009) } \\
*\end{array}$ & $\begin{array}{l}\text { Berücksichtigung d. } \\
\text { U.-kontext }\end{array}$ & $\begin{array}{l}\text { Einzelfallstudie } \\
\text { in KMU }\end{array}$ & Microblogging & 2,3 \\
\hline $\begin{array}{l}\text { Buhse und } \\
\text { Stammer } \\
(2009)\end{array}$ & $\begin{array}{l}\text { Freiwilligkeit und } \\
\text { Kontrolle, Nutzer- } \\
\text { beteiligung, u.a. }\end{array}$ & $\begin{array}{l}\text { Ca. } 5 \text { Einzelfall- } \\
\text { studien, U. aller } \\
\text { Größen }\end{array}$ & Wiki, Weblog & $\begin{array}{l}1,2, \\
4,5\end{array}$ \\
\hline $\begin{array}{l}\text { Ebersbach } \\
\text { und Glaser } \\
(2009)^{*}\end{array}$ & $\begin{array}{l}\text { Nutzerrollen und- } \\
\text { gruppen, Wiki als } \\
\text { Suchportal, u.a. }\end{array}$ & $\begin{array}{l}\text { Einzelfallstudie: } \\
\text { IBM }\end{array}$ & Wiki & 3,5 \\
\hline $\begin{array}{l}\text { Happel und } \\
\text { Treitz } \\
(2008)\end{array}$ & $\begin{array}{l}\text { Wiki-Wucherung / } \\
\text { Qualitätssicherung }\end{array}$ & $\begin{array}{l}\text { Mehrfachfallstu- } \\
\text { die in } 6 \mathrm{U} \text {. }\end{array}$ & Wiki & 6 \\
\hline $\begin{array}{l}\text { Koch und } \\
\text { Richter } \\
(2009)^{*}\end{array}$ & $\begin{array}{l}\text { Ziele, Anwendungs- } \\
\text { fälle und -muster, } \\
\text { freudvolle Nutzung, } \\
\text { Motivation, u.v.a. }\end{array}$ & $\begin{array}{l}21 \text { Einzelfallstu- } \\
\text { dien in Unter- } \\
\text { nehmen aller } \\
\text { Größen }\end{array}$ & $\begin{array}{l}\text { Wiki, Weblog, } \\
\text { Social Tagging, } \\
\text { SNS }\end{array}$ & $\begin{array}{l}1,2, \\
3,4, \\
5,6\end{array}$ \\
\hline $\begin{array}{l}\text { Linderman } \\
\text { n et al. } \\
(2009)\end{array}$ & $\begin{array}{l}\text { Anforderungen an } \\
\text { eine E2.0-Plattform }\end{array}$ & $\begin{array}{l}\text { Mehrfachfallstu- } \\
\text { die in } 6 \mathrm{KMU}\end{array}$ & SNS & $\begin{array}{l}3,4, \\
5,6\end{array}$ \\
\hline $\begin{array}{l}\text { Müller und } \\
\text { Dibbern } \\
(2006)^{*}\end{array}$ & $\begin{array}{l}\text { Einführungsprozess, } \\
\text { Erforderliche In- } \\
\text { stanzen, u.a. }\end{array}$ & $\begin{array}{l}\text { Einzelfallstudie } \\
\text { in KMU }\end{array}$ & Wiki & 3,5 \\
\hline $\begin{array}{l}\text { Räth et al. } \\
(2009)\end{array}$ & $\begin{array}{l}\text { U.-kultur, Akzep- } \\
\text { tanz, u.a. }\end{array}$ & $\begin{array}{l}\text { Mehrfachfallstu- } \\
\text { die in KMU }\end{array}$ & Weblog & 2,5 \\
\hline $\begin{array}{l}\text { Richter und } \\
\text { Koch } \\
(2009) *\end{array}$ & $\begin{array}{l}\text { Datenschutz, Tool- } \\
\text { integration, Medi- } \\
\text { enwahl, u.v.a. }\end{array}$ & $\begin{array}{l}\text { Einzel- und } \\
\text { Mehrfachfallstu- } \\
\text { die in } 3 \mathrm{U} \text {. }\end{array}$ & SNS & $\begin{array}{l}3,4, \\
5,6\end{array}$ \\
\hline $\begin{array}{l}\text { Schachner } \\
\text { und Toch- }\end{array}$ & $\begin{array}{l}\text { „Dominanz von } \\
\text { Daten“, Nutzerbe- }\end{array}$ & $\begin{array}{l}5 \text { Einzelfallstudi- } \\
\text { en in U. aller }\end{array}$ & Wiki, Weblog & $\begin{array}{l}1,2, \\
5\end{array}$ \\
\hline
\end{tabular}




\begin{tabular}{|l|l|l|l|l|}
\hline Quelle & $\begin{array}{l}\text { Betrachtete } \\
\text { Themata }\end{array}$ & $\begin{array}{l}\text { Empirische } \\
\text { Basis }\end{array}$ & $\begin{array}{l}\text { Anwendungs- } \\
\text { klasse }\end{array}$ & $\begin{array}{l}\text { Me- } \\
\text { ta }\end{array}$ \\
\hline $\begin{array}{l}\text { termann } \\
(2008)\end{array}$ & $\begin{array}{l}\text { teiligung, U.- kon- } \\
\text { text, u.a. }\end{array}$ & größen & & \\
\hline $\begin{array}{l}\text { Stein und } \\
\text { Blaschke } \\
(2009)\end{array}$ & $\begin{array}{l}\text { Möglichkeiten der } \\
\text { Datenvisualisierung }\end{array}$ & $\begin{array}{l}\text { Komp. Daten- } \\
\text { analyse in 4 U. }\end{array}$ & Wiki & 2,6 \\
\hline $\begin{array}{l}\text { Stocker } \\
(2010)^{*}\end{array}$ & $\begin{array}{l}\text { Akzeptanz, U.- } \\
\text { kultur, Interaktions- } \\
\text { typen, u.v.a. }\end{array}$ & $\begin{array}{l}\text { Mehrfachfallstu- } \\
\text { die mit 10 U. } \\
\text { aller Größen }\end{array}$ & Wiki, Weblog & $\begin{array}{l}2,4, \\
5,6\end{array}$ \\
\hline $\begin{array}{l}\text { Warta } \\
(2009)^{*}\end{array}$ & $\begin{array}{l}\text { Einfluß der Wiki- } \\
\text { Engine, Informati- } \\
\text { onsqualität, u.a. }\end{array}$ & $\begin{array}{l}\text { Komparative } \\
\text { Datenanalyse mit } \\
13 \text { Konzernen }\end{array}$ & Wiki & $\begin{array}{l} \\
5,3, \\
5,6\end{array}$ \\
\hline
\end{tabular}

\section{Datenerhebung}

Die Datenbasis des Beitrags bildet ein eintägiger Workshops zum Thema Enterprise 2.0, an welchem 19 Experten aus Wissenschaft und Praxis, die in diesem Bereich arbeiten und forschen, teilnahmen. Der Workshop wurde über die gesamte Dauer von den beiden Koautoren und einem unabhängigen Experten begleitet. Einer der beiden Koautoren nahm als Moderator an dem Workshop teil, der andere sowie der unabhängige Experte waren als teilnehmender Beobachter anwesend.

Der Workshop war wie folgt gegliedert: Impulsreferate und jeweils kurze Diskussion zu aktuellen Forschungsprojekten und -schwerpunkten der Experten (90 Minuten), moderierte Plenumsdiskussion zur Identifikation besonders relevanter Themenbereiche im Forschungs- und Praxiseinsatzfeld Enterprise 2.0 (90 Minuten), vertiefte thematische Diskussion in Kleingruppen (jeweils 3 bis 4 Personen) zur Detaillierung eines Themenbereichs (90 Minuten), Präsentation der Ergebnisse der Kleingruppendiskussion und moderierte Plenumsdiskussion zu den erarbeiteten Themenfeldern (90 Minuten). Mithilfe von Notizen, Photoprotokollen und den erstellten Postern wurde der Workshop von den Koautoren dokumentiert.

Das Ergebnis des Workshop und der anschließenden Analyse durch die Autoren ist eine Forschungsagenda mit sechs Metathemata, welche in der nachfolgenden Abbildung überblicksartig dargestellt sind: Zielsetzung \& Definition, Enterprise 2.0 in der Organisation, Funktionalitäten, Motivation, Nutzung und Daten ${ }^{5}$.

\footnotetext{
${ }^{5}$ Die sechs Bereiche sind logisch gereiht; eine Priorisierung wird durch diese Reihenfolge nicht explizit vorgenommen. Ebenso wenig ist es das Ziel der Abbildung Verknüpfungen oder Überschneidungen der einzelnen Themenfelder darzustellen. Diese können zwischen allen Bereichen auftreten.
} 


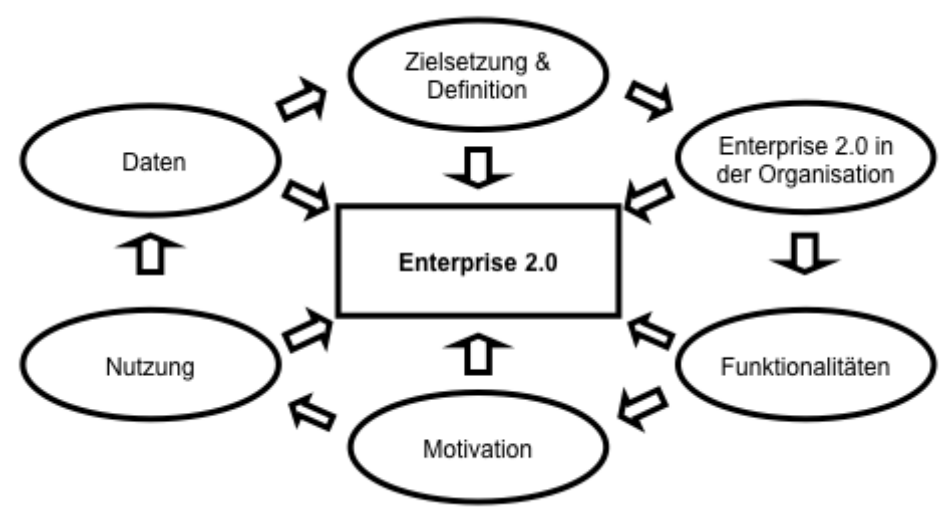

Abbildung 1: Metathemata im Bereich Enterprise 2.0

\section{Ergebnisse: Zukunft von Enterprise 2.0}

Nachfolgend werden die erarbeiteten sechs Metathemata vorgestellt, in welchen zukünftige Forschungen zum Themengebiet Enterprise 2.0 als besonders vordringlich erscheinen.

\subsection{Metathema 1: Zielsetzung \& Definition}

Das Metathema 1 umreißt erstens den Bedarf einer Festlegung, was unter dem Begriff „Enterprise 2.0“ generisch zu verstehen ist, um von dieser gemeinsamen Grundlage aus Deutungen für den Einzelfall vornehmen zu können. Hierbei erscheint wesentlich, dass eine ausdifferenzierte, allgemeingültige Definition weder notwendig noch tatsächlich hilfreich ist, sondern vielmehr ein kleinster gemeinsamer Nenner gefunden werden soll, auf welchem aufbauend Definitionen für spezielle Anwendungen oder Forschungsfragen erarbeitet werden können.

Zweitens verbalisiert es die Notwendigkeit, generisch wie individuell die Zielsetzungen zu präzisieren, welche mithilfe des Einsatzes von Social Software im Unternehmen erreicht werden sollen. Ein modularer Aufbau scheint hier vorteilhaft, da die Zielsetzungen naturgemäß zwischen den implementierenden Organisationen divergieren.

$(\mathrm{L})^{6}:$, Wenn ich an einen Mittelständler denke, wird dieser doch zwangsläufig ganz andere Vorstellungen und Erwartungen haben als ein Großunternehmen. Für den ist ein Blog doch schon Enterprise 2.0.“

${ }^{6}$ Es handelt sich jeweils um (anonymisierte) Aussagen der Teilnehmer des Workshops. 
Eine wissenschaftliche Ausarbeitung verschiedener Zielsetzungen inklusive prioritären Handlungsanleitungen, welche aus den Zielsetzungen resultieren, erscheint aus wissenschaftlicher wie praktischer Hinsicht dringend notwendig. Wesentlich erschien den Experten zudem, dass im Zusammenhang mit Zielsetzungen nicht nur zentrale Erfolgskriterien monetärer und prozessualer Art definiert, sondern auch soziale Protokolle in den Zielkatalog aufgenommen werden. Hierzu sagte einer der Teilnehmer:

(M): „Es geht ja bei Enterprise 2.0 gerade um das Verhalten der Nutzer - welche sozialen Protokolle werden angelegt, wie und warum wird sanktioniert oder belohnt.“

\subsection{Metathema 2: Enterprise 2.0 in der Organisation}

Beim Metathema 2 erachteten die Experten insbesondere die Auswirkungen bestehender Systeme auf neu einzuführende („Verhältnis von Enterprise 1.0 zu Enterprise 2.0") für wichtig, welche über die funktionale und technische Seite hinaus ernstzunehmende Wechselwirkungen mit den organisationalen Aspekten einer Unternehmung haben. Dazu kommt, dass der Einsatz von Social Software zunehmend weniger prototypisch abläuft, und den Weg in die Arbeitsprozesse findet („Enterprise 2.0 gets serious"). Daraus ergeben sich Fragestellungen nach dem Zusammenspiel verschiedener Einflußfaktoren wie z.B. der Unternehmensgröße oder -kultur. Zudem ist ein Wandel in der Wahrnehmung der Dienste zu bemerken:

(N): „Man muss sich das mal vorstellen: Da sind die ganzen Daten in einem Blog gespeichert und sonst nirgendwo. Keiner hat ein Backup und wenn es abstürzt, ist alles weg."

\subsection{Metathema 3: Funktionalitäten}

Aufgrund der stetig anwachsenden Anzahl von Anwendungssystemen, welche unter den Begriff Enterprise 2.0 fallen, zeigt sich im Rahmen des Metathema 3 v.a. aus Praxissicht die Notwendigkeit einer Typologisierung der Funktionen. Diese sollte möglichst flexibel angelegt werden, damit Veränderungen rasch dargestellt werden können:

(E): „Eine Typologisierung ist zwanghaft veraltet, wenn sie nicht ausreichend flexibel angelegt ist, um zum Beispiel die Integration von Instrumenten - wenn das Wikisystem plötzlich soziale Vernetzung erlaubt, indem auch die Autoren des Beitrags angezeigt werden - adäquat darzustellen!“

Eine Typologisierung könnte ebenso die Nutzer bei ihrer Medienwahl unterstützen und die Verwirrung angesichts der Vielfalt mindern: 
(Ä): „Der Punkt ist doch, dass ich vor lauter Tools schon gar nicht mehr weiß, was ich jetzt twittern, bloggen oder aufs Wiki stelle. Das ist ja eine wahre Tooleritis.“

Zudem ist ein Trend zu immer schlankeren Anwendungen auszumachen, der auch dazu führt, dass die Systeme zunehmend zusammenwachsen („Vermash(up)ung ${ }^{\circ}$ ). Hierbei stellt sich auf technischer Seite die Frage nach sinnvollen Integrationskonzepten. Bei deren Entwicklung sollten jedoch weniger die technisch möglichen Funktionalitäten, sondern vielmehr die gewünschten und benötigten Eigenschaften (,Simplicity “) der Anwendungen im Vordergrund stehen, um die Nutzungsbarrieren zu senken:

(M): „Wir müssen aufhören, immer nur an die Machbarkeit zu denken. Wesentlich ist doch, was die Nutzer brauchen, wozu die Anwendung eingesetzt werden soll und warum. Das muss die Entwicklung treiben.“

\subsection{Metathema 4: Motivation}

Auch beim Einsatz von Social Software in Unternehmen spielt - wenn auch differenziert zum freiwilligen Web 2.0 - der Faktor Motivation eine wichtige Rolle. Hierbei sind Fragen zu beantworten, wie man initial eine kritische Masse von Benutzern erreicht und wie die Beteiligung hoch gehalten werden kann. So stellte zum Beispiel ein Teilnehmer den Nutzen von "Lead Usern" in Frage:

$(\mathrm{H})$ : „Wenn ich nun die Nutzer der ersten Stunde in einem Newsletter explizit erwähne, freuen die sich vielleicht - die anderen sehen das aber möglicherweise als abschreckend an oder halten die Aufgabe für gelöst.“

In diesem Zusammenhang stellen sich daher Fragen nach dem Einsatz geeigneter Anreizsysteme, aber auch danach wie weit die für Social Software typische Freiwilligkeit und Selbstorganisation gehen kann. Bereits mehrfach untersucht, doch noch immer ungelöst, ist diesbezüglich die ungleiche Beitragsverteilung zwischen Nutzern.

(G): „Es stellt sich die Frage, ob das Attribut „Freiwilligkeit“ noch gültig ist, wenn ich meine Mitarbeiter mehrfach explizit auffordere, sich an dem Wiki zu beteiligen oder dies sogar in ihre Zielvereinbarung aufnehme."

\subsection{Metathema 5: Nutzung}

Die in Metathema 4 identifizierte Herausforderung ungleicher Beitragsverteilung kann zur Identifikation verschiedener Nutzergruppen oder auch „Interaktionstypen" genutzt werden. So beschreibt eine Gruppe von Teilnehmern: 
(Gruppe Meta 4): „Man muss da zwischen der Person und der Rolle, welche sie in einem Kooperationssystem spielt, unterscheiden. Das Nutzungsverhalten ist keineswegs nur freiwillig und persönlich getrieben.“

Die Klassifikation der Nutzergruppen kann desweiteren den Verantwortlichen für die Nutzung Hilfestellung bieten, um die Medienkompetenz der Nutzer weiter zu erhöhen und $\mathrm{zu}$ verhindern, dass es $\mathrm{zu}$ einer sogenannten digitalen Spaltung kommt. Dies wurde wie folgt zusammengefasst:

(M): „Es ist wesentlich, dass erkannt wird, wer welche Kompetenzen im Umgang mit den Anwendungen hat und braucht."

\subsection{Metathema 6: Daten}

Schließlich findet in Metathema 6 ein besonderer Aspekt von Social Software Beachtung: Aufgrund der hohen Beteiligung der Nutzer liegen in der Regel eher zu viele als zu wenige Daten vor. Damit verbunden ist zunächst einmal die Frage, ob es sich bei Enterprise 2.0 nicht oftmals um ein „Informationsrauschen“" handelt, das die Aufmerksamkeit an die falschen Stellen lenkt und auch, ob es sinnvoll oder gar notwendig geworden ist eine Art „Informations- bzw. Relevanzfilterung“ vorzunehmen. Ein Teilnehmer formulierte die Herausforderung so:

(E): „Mein Tag hat einfach nur 24 Stunden. Will ich da wirklich die 17 Updates eines Kollegen aus einer anderen Fachabteilung lesen? Sind für mich die letzten 5 Änderungen auf dem Wiki relevant? Wie kann ich das filtern?““

In diesem Zusammenhang wurde auch die Auswertung der zahlreichen nutzergenerierten Daten sehr kontrovers diskutiert. Diese stößt zum einen oftmals auf Widerstände in den Mitarbeitervertretungen, da noch Lösungen zum Schutz persönlicher und/oder sensibler Daten gefunden werden müssen. Andererseits steht die Auswertung der Daten üblicherweise nur dem Management zur Verfügung, was technisch keinesfalls notwendig ist. Allerdings würde eine Öffnung der Auswertungsfunktion kulturell und organisatorisch $\mathrm{zu}$ wesentlichen Veränderungen führen, deren Folgen noch nicht abschätzbar sind. Eine Gruppe von Teilnehmern schlug dennoch vor:

(Gruppe Meta 6): „Warum werden die Daten nicht für alle geöffnet, die sich dafür interessieren? Alle Auswertungen werden allen Anwendern ermöglicht. Das wäre doch ein faire Herangehensweise." 


\section{Zusammenfassung und Ausblick}

Der vorliegende Beitrag zeigt, basierend auf der Literaturanalyse und der Fokusgruppe mit Experten, die Vielseitigkeit der Themenfelder im Bereich Enterprise 2.0 auf und systematisiert den aktuellen Stand der Erkenntnis. Wir zeigen sechs Metathemen auf, in welchen Forschungsbedarf für Wissenschaft und Praxis im Feld Enterprise 2.0 besteht. Jedes dieser Metathemata - Zielsetzung und Definition, Enterprise 2.0 in der Organisation, Funktionalitäten, Motivation, Nutzung und Datenverdient für sich genommen die Aufmerksamkeit von Forschung und Praxis. Allerdings erachten wir auch die Verbindung verschiedener Metathemata für relevant, da diese, wie in Abbildung 1 dargestellt, miteinander in Beziehung stehen und sich gegenseitig beeinflussen. Beispielsweise sind der Zusammenhang zwischen Enterprise 2.0 in der Organisation und der Umgang mit Daten eng verbunden. Die Metathemata wurden in Tabelle 1 (in Spalte „Meta“) den in Überblick berücksichtigten Studien zu Enterprise 2.0 zugeordnet. Wie ersichtlich ist die einzelne Betrachtung eines Metathemas eher die Ausnahme, sondern es werden stets mehrere Metathemata beobachtet. Daraus schließen wir, dass sich ein großer Teil der deutschsprachigen Studien eher darauf konzentriert, ein ganzheitliches Bild zu vermitteln, anstatt sich auf einzelne Themenbereiche zu fokussieren.

Die vorgeschlagene Forschungsagenda der sechs Metathemata muss im Licht der Limitationen des vorliegenden Beitrags betrachtet werden. So stellt die Beschränkung sowohl des Literaturüberblicks als auch der Fokusgruppe auf deutschsprachige Beiträge und Experten sicherlich ein Hindernis zur Verallgemeinerung der Ergebnisse dar. Zukünftige Arbeiten im Feld sollten daher die vorgeschlagenen Metathemata auf ihre Gültigkeit überprüfen, indem ein systematischer Literaturüberblick auch im englischsprachigen Bereich durchgeführt und ein noch größeres Feld aus Experten verschiedener Nationen befragt wird. Eine vergleichende Analyse der Ergebnisse lässt sowohl für das Forschungsfeld wie auch für die Praxis wesentliche Erkenntnisse erwarten.

\section{Danksagung}

Wir möchten uns hiermit herzlich bei allen Teilnehmern des Workshops bedanken: Martin Böhringer, Karsten Ehms, Peter Geißler, Markus Glaser, Markus Heckner, Hendrik Kalb, Susanne Mörl, Christian Neubert, Christian Reuter, Michael Stecher, Martin Wünnenberg. Nadine Lindermann, Melanie Steinhüser und Michael Koch haben uns darüber hinaus wertvolle Hinweise gegeben, für die wir zusätzlich danken möchten. Die Realisierung des Projekts wurde ermöglicht durch die Förderung des Bundesministeriums für Bildung und Forschung und des Europäischen Sozialfonds (BALANCE von Flexibilität und Stabilität in der Forschungswelt, FKZ 01FH09153 und GENIE: Gemeinschaftsgestützte Innovationsentwicklung für Softwareunternehmen, FKZ 01FM07029). 


\section{Literatur}

Back A, Gronau N, Tochtermann K (2009) Web 2.0 in der Unternehmenspraxis. Oldenbourg, München.

Benlian A, Hilkert D, Hess T (2009) eCollaboration mit Social Software in der globalen Softwareentwicklung. In: HMD - Praxis der Wirtschaftsinformatik, 267:37-46.

Blaschke S (2008) Wikis in Organisationen: Von Kommunikation zu Kollaboration. In: Alpar P, Blaschke S (Hrsg.) Web 2.0 - Eine empirische Bestandsaufnahme. Teubner+Vieweg, Wiesbaden:183-203.

Böhringer M, Koch, M., Richter, A. (2009) Awareness 2.0 - Ein Anwenderbeispiel von Microblogging im Unternehmen. In: Information Wissenschaft \& Praxis, 60(4):275-279.

Buhse W, Stamer S (2008) Enterprise 2.0 - Die Kunst, loszulassen. RhombosVerlag, Berlin.

BITKOM (2007) ITK im Mittelstand zur Erhöhung der Wettbewerbsfähigkeit Analyse zum Informationsbedarf in mittelständischen Unternehmen. http://www.bitkom.org/files/documents/ ITKBedarfsanalyse_Mittelstand.pdf. (alle Online-Quellen wurden im Sept. 09 noch einmal geprüft)

Bughin J, Manyika J (2007) How businesses are using Web 2.0. In: McKinseyQuarterly 3/2007.

Bughin J, Manyika J, Miller A (2008) Building the Web 2.0 Enterprise. In: McKinsey Quarterly 7/2008.

Bughin J, Manyika J, Miller A (2009) How companies are benefiting from Web 2.0. In: McKinsey Quarterly 9/2009.

Berlecon Research (2007) Enterprise 2.0 in Deutschland - Verbreitung Chancen und Herausforderungen. http://www.coremedia.com/de/124518/studie/.

Döbler T (2007) Potenziale von Social Software. MFG Stiftung BW Stuttgart. http://www.fazit-forschung.de/fileadmin/_fazitforschung/downloads/FAZIT_Schriftenreihe_Band_5.pdf

Ebersbach A, Glaser M (2009) Wiki als zentrales Suchportal. Das Beispiel bluepedia. In: Information Wissenschaft und Praxis, (60)4, 197-201. 
Fuchs-Kittowski F, Klassen N, Faust D, Einhaus J (2009) A Comparative Study on the Use of Web 2.0 in Enterprises. In: Proceedings 9th International Conference on Knowledge Management and Knowledge Technologies, Graz.

Granitzer G, Tochtermann K. (2009) Web 2.0 in Unternehmen - Eine

Fallstudienanalyse. In: Proceedings 5. Konferenz Professionelles

Wissensmanagement, Solothurn.

Happel H, Treitz M (2008) Proliferation in Enterprise Wikis. In: Proceedings of the 8th International Conference on the Design of Cooperative Systems (COOP'08).

Herrmann H.-G. (2007) Internet und Intranet 2.0 - Chancen und Risiken für Großkonzerne In: OSCAR 2/2007:10-16.

Jahnke I (2009) Socio-technical Communities: From Informal to Formal? In:

Withworth B (Hrsg.): Handbook of Research on Socio-Technical Design and Social Networking Systems. IGI Global Publisher.

Koch M, Richter A (2009) Enterprise 2.0 - Planung Einführung und erfolgreicher Einsatz von Social Software im Unternehmen, Oldenbourg, München.

Leibhammer J, Weber M (2008) Enterprise 2.0. Analyse zu Stand und Perspektiven in der deutschen Wirtschaft.

http://www.bitkom.org/files/documents/BITKOM-

Studie_Enterprise_2Punkt0.pdf.

Lindermann N, Valcárcel S, Abram I, Blinn N, Fäcks K, Jung RH, von

Kortzfleisch HFO, Nüttgens M (2009) Netzwerken 2.0 in KMUs - Kleine und mittlere Unternehmen im Zentrum Web 2.0 basierter Kooperation.

Arbeitsberichte aus dem Projekt KMU 2.0, 1/2009.

McAfee A (2006) Enterprise 2.0 - The Dawn of Emergent Collaboration. In: MIT Sloan Management Review 47(3):21-28.

Müller C, Dibbern P (2006) Selbstorganisiertes Wissensmanagement in

Unternehmen auf Basis der Wiki-Technologie - ein Anwendungsfall. In: HMD

- Praxis der Wirtschaftsinformatik 252:45-54.

Räth P, Schwaab J, Smolnik S, Urbach N (2009) Weblogs in der internen

Zusammenarbeit der GTZ. HMD - Praxis der Wirtschaftsinformatik 267:2736.

Richter A, Koch M (2009) Der Einsatz von Social Networking Services im

Unternehmen. In: Proceedings Internationale Konferenz

Wirtschaftsinformatik, Wien.

Schachner W, Tochtermann K (2008) Corporate Web 2.0 - Band 2. Shaker Verlag, Aachen. 
Stein K, Blaschke S (2009) Corporate Wikis: A Comparative Analysis of Structures and Dynamics. In: Proceedings 5. Konferenz Professionelles Wissensmanagement, Solothurn.

Stocker A (2009) Intraorganisationaler Wissenstransfer über Wikis und Weblogs. Dissertation, Karl-Franzens-Universität Graz. (wird veröffentlicht)

T-Systems (2008) Trendsurvey: Enterprise 2.0 erobert Unternehmen. http://www.t-systems-mms.com/workplace

Warta A (2009) Indikatoren für den Erfolg von Unternehmenswikis. Dissertation, Universität Konstanz. (wird veröffentlicht)

Wittmann G, Werner C, Krabichler T, Stahl E (2008) Web-2.0 bei Finanzdienstleistern - Auf halber Strecke Richtung Zukunft. Arbeitsbericht, ibi research Regensburg.

Zerfaß A, Welker M, Schmidt J (2008) Kommunikation, Partizipation und Wirkungen im Social Web. Herbert von Halem Verlag, Düsseldorf. 